\title{
Alitiasic Cholecystitis and Acute Pancreatitis Secondary to Gastroduodenal Intussusception after Roux-En-Y Gastric Bypass Case Report
}

\section{Alberto Perez Castilla A* and Penailillo P \\ Department of Digestive Surgery, Indisa Clinic. Santiago, Chile}

*Corresponding author: Perez Castilla A, Department of Digestive Surgery, Clinica Indisa, University Andres Bello, Santa Maria 1810 Providencia, Santiago, Chile, Tel: 56954111154; Email: investigacionindisa@gmail.com

\section{Case Report \\ Volume 2 Issue 3}

Received Date: August 14, 2018

Published Date: October 03, 2018

\section{Abstract}

Obesity today is a public health problem in most industrialized countries, Chile does not escape this reality. Despite the availability of medical treatments and changes in lifestyle, bariatric surgery is a safe and reliable therapeutic alternative for long-term treatment. However, it is not free of risks or complications. Intussusception is a complication that must be suspected, although its reportad incidence is close to $0.1-0.3 \%$. In this Report, we describe an un usual case in a patient presenting acute alitiasis cholecystitis and acute pancreatitis secondary to gastric intussusception in the duodenum, 4 months after surgery for Roux-en-Y Gastric Bypass.

Keywords: Bariatric surgery; Roux-en-Y Gastric Bypass; Alitiasic Cholecystitis; Colic abdominal pain; Laparoscopy

\section{Introduction}

Obesity today is a public health problem in most industrialized countries, Chile does not escape this reality [1]. Despite the availability of medical treatments and changes in lifestyle, bariatric surgery is a safe and reliable therapeutic alternative for long-term treatment. However, it is not free of risks or complications. In the universe of complications after surgery, diagnosis and early management reduce the morbidity and mortality of patients. Intussusception is a complication that must be suspected, although its reportad incidence is close to 0.1$0.3 \%$ [2]. Clear diagnostic and management guidelines should be applied, contrast-enhanced abdominal CT being the most efficient means to confirm the presence of intussusception, since the clinic usually does not provide sufficient elements to establish the diagnosis [2-4]. Of the cases reportad today, jejunal-jejunoanastomosis intussusception is the usual presentation. In this report, we describe an un usual case in a patient presenting acute alitiasis cholecystitis and acute pancreatitis secondary to gastric intussusception in the duodenum, 4 months after surgery for Roux-en-Y Gastric Bypass.

\section{Case}

A 43-year-old female patient comes to the emergency department with a history of 1 month of intermittent colic abdominal pain, with a predominance of upper hemiabdomen. Her previous medical history included hypertension, insulin resistance syndrome, smoking and obesity, having undergone 4 months prior to correction 
surgery of Hiatal Hernia and Laparoscopic Roux-en-Y Gastric Bypass. Preceding to its consultation, EED had been carried out, which reportad adequate transit of contrast from gastric pouch to jejunal loop, with a smaller caliber, partially distensible gastrojejunal anastomosis, distal jejunal loops and ileon that adequately opacified with preserved peristalsis.

Physical examination show soft abdomen with mild sensitivity in the right hypochondrium. Routine tests reporting WBC $8.7 \mathrm{~K} / \mathrm{dL}(\mathrm{nl} 4.1$ - 9.5) Amylase $305 \mathrm{U} / \mathrm{L}$ (nl 30 - 110) Lipase 2582 U/L (nl 23-300) Total bilirubin $0.20 \mathrm{mg} / \mathrm{dL}$ (Nl 0.2-1.3) PCR $0.90 \mathrm{mg} / \mathrm{dL}$ (nl 0-1). The remaining exams are close to normal. It was request abdominal ecotomography that reports biliary mud. Regarding antecedents and clinical presentation, it was order an abdominal CT with EV contrast that reports dilatation of intra and extrahepatic biliary tract, to the intrapancreatic portion, with slight dilatation of the main pancreatic duct, Gastroduodenal invagination up to the level of jejuno-jejunostomy that causes extrinsic compression of the invaginated content at the level of the duodenal papilla and the head of the pancreas (Figure 1). We performed exploratory laparoscopy, identifying the stomach completely intussuscepted in the duodenum (Figure 2). The stomach is removed from the duodenum, with protrusion of suture, with a normal-looking duodenum. Therefore, a remnant gastrectomy is performed, removing major and minor omentum, release of duodenum and post-pyloric section. Since the patient presented distended gallbladder with edematous tension, and dilated biliary tract, intraoperative cholangiography is performed without evidence of choledocholithiasis, so a cholecystectomy is perform without incidente. It begins to drink liquids in 24 hours and 5 days after the surgical procedure is discharge. Biopsy of the stomach reported granulatory tissue, cicatrizal fibrosis, chronic granulomatous inflammation, multinucleated giant cells, reaction to foreign bodies, mild superficial chronic gastritis and duodenal wall segment without morphological alterations. The anatomopathological study of the gallbladder indicated moderate chronic cholecystitis, cholesterolosis and cystic ganglion with reactive lymphadenitis.

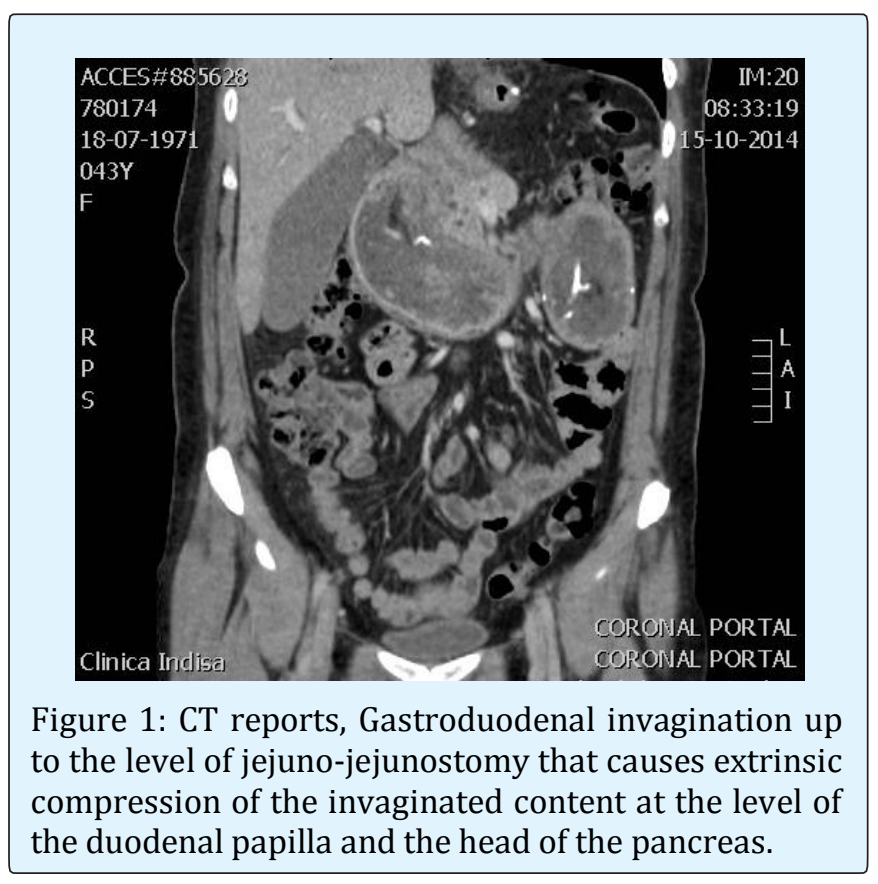

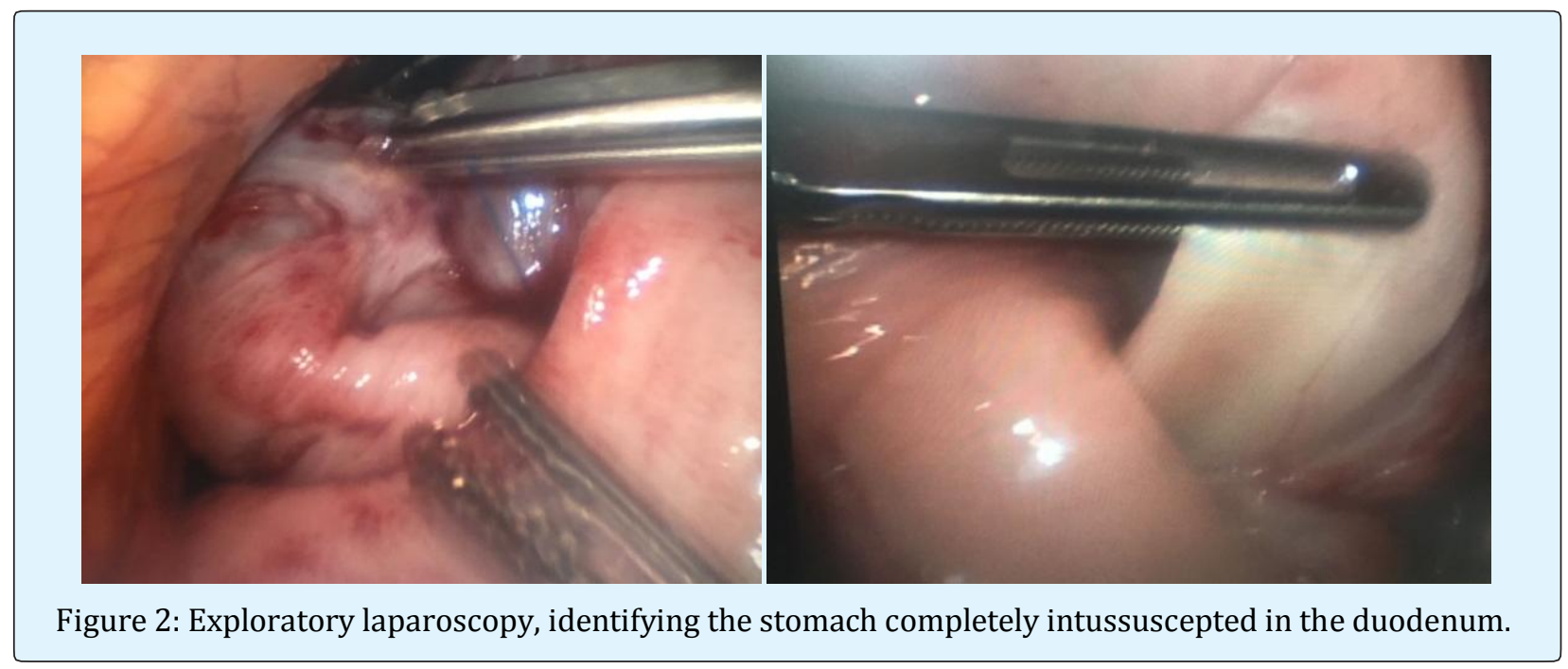

Alberto Perez Castilla A and Penailillo P. Alitiasic Cholecystitis and Acute Pancreatitis Secondary to Gastroduodenal Intussusception after Roux-En-Y Copyright@ Alberto Perez Castilla A and Penailillo P. Gastric Bypass Case Report. Int J Surg Surgical Tech 2018, 2(3): 000128. 


\section{International Journal of Surgery \& Surgical Techniques}

\section{Discussion}

Intussusception as a complication of bariatric surgery is relatively uncommon [2,5] however, with newest techniques, current obesity statistics and hence the increased frequency of this surgical treatment, the increase in the incidence of this or other complications is expected. It is evident that the majority of the affected patients are female, since they are the group that most undergo bariatric procedures [3]. Of the reported cases of intussusceptions, they commonly result from the retrograde type, with the majority being invagination through the jejuno-jejunostomy $[2,3]$.

In this particular case, the symptomatology is nonspecific, short duration, more compatible with pancreatitis and acute cholecystitis due to both the symptoms and the laboratory results [6]. Therefore it was decided to request imaging studies that complemented the diagnosis, being the abdomen CT scan the most specific to establish the etiology. The presence of intussusception should always be suspected in a bariatric patient, especially when the clinic is non-specific and the presence of recurrent symptomatology [2-5]. The management of the case was to modify the main complication without disregarding its concomitant pathologies: correct the intussusception [5] and manage the inflammatory pancreaticobiliar signs $[6,7]$. In this patient, since the affected organ was the gastric remnant, the surgical procedure consisted of gastrectomy and additionally cholecystectomy plus intraoperative cholangiography [8]. The surgical resolution should not be postponed, since waiting increases morbi-mortality.

\section{Conclusion}

Intussusception in patients with bariatric surgery, although infrequent, should be suspected in the case of non-specific, recurrent abdominal pain, nausea and vomiting. The most reliable imaging method for diagnosis is CT scan of the abdomen with contrast. Surgical behavior will depend on the viability of the affected segment and should not be postponed because of diagnostic accuracy. Surgical management in the first instance should be laparoscopic. The behavior in case of gastric intussusception is resection.

\section{References}

1. Minsal Departamento de estadísticas e información en Salud.

\section{2. http://www.deis.cl/?s=minsal}

3. Simper SC, Erzinger JM, McKinlay RD, Smith SC (2008) Retrograde (reverse) jejunal intussusception might not be such a rare problem: a single group's experience of 23 cases. Rocky Mountain Associated Physicians, Salt Lake City, Utah. Surgery for Obesity and Related Diseases 4 (2): 77-83.

4. Singla S, Guenthart AB, LaurenMay, John Gaughan, Meilahn JE (2012) Intussusception after Laparoscopic Gastric Bypass Surgery: An Underrecognized Complication.. Department of Surgery, Temple University Hospital. Hindawi Publishing Corporation Minimally Invasive Surgery pp: 8.

5. Maghrebi H, Makni A, Rhaiem R, Atri S, Ayadi M, et al. (2017) Adult intussusceptions: Clinical presentation, diagnosis and therapeutic management. Surgery Department La Rabta Hospital, Tunis, Tunisia; Faculty of Medicine of Tunis, University of Tunis El Manar, Tunisia Int J Surg Case Rep 33: 163-166.

6. Oliver Varban, Ali Ardestani, Dan Azagury, David B. Lautz, Ashley H. Vernon, et al. (2013) Resection or reduction? The dilemma of managing retrograde intussusception after Roux-en-Y gastric bypass. Department of Surgery, Brigham and Women's Hospital, Harvard Medical School, Boston, Massachusetts. Surgery for Obesity and Related Diseases 9 (5): 725-730.

7. Arapis K, Tammaro P, Goujon G, Becheur H, Augustin $P$, et al. (2017) Elevated plasma pancreatic enzyme concentrations after Roux-en-Y gastric bypass may indicate closed loop obstruction, Bichat-Claude Bernard Hospital, Paris, France. Ann R Coll Surg Engl 2017 99(2): e62-e64.

8. Pande R, Fraser I, Harmston C (2012) Emergency presentation of retrograde intussusception as a late complication of gastric Bypass. University Hospitals Coventry and Warwickshire NHS Trust, UK Ann R Coll Surg Engl 94(3): 116-117.

9. Bradley JF, Ross SW, Christmas AB, Fischer PE, Sachdev G, et al. (2015) Complications of bariatric surgery: the acute care surgeon's experience. Department of General Surgery Carolinas Medical Center, Charlotte, NC, USA. The American Journal of Surgery 210(3): 456-461. 


\section{International Journal of Surgery \& Surgical Techniques}

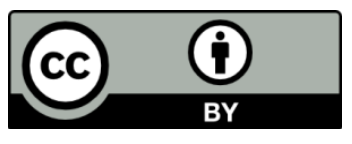

